\title{
A note on the diophantine equation $x^{2}+b^{y}=c^{z}$
}

by

MaOhuA Le (Zhanjiang)

1. Introduction. Let $\mathbb{Z}, \mathbb{N}, \mathbb{Q}$ be the sets of integers, positive integers and rational numbers respectively. Let $(a, b, c)$ be a primitive Pythagorean triple such that

$$
a^{2}+b^{2}=c^{2}, \quad a, b, c \in \mathbb{N}, \operatorname{gcd}(a, b, c)=1,2 \mid a .
$$

Then we have

$$
a=2 s t, \quad b=s^{2}-t^{2}, \quad c=s^{2}+t^{2},
$$

where $s, t \in \mathbb{N}$ satisfy $\operatorname{gcd}(s, t)=1, s>t$ and $2 \mid s t$. Recently, Terai [5] conjectured that the equation

$$
x^{2}+b^{y}=c^{z}, \quad x, y, z \in \mathbb{N},
$$

has only the solution $(x, y, z)=(a, 2,2)$. Simultaneously, he proved that if $b \equiv 1(\bmod 4), b^{2}+1=2 c, b, c$ are odd primes, $c$ splits in the imaginary quadratic field $K=\mathbb{Q}(\sqrt{-b})$ and the order $d$ of a prime ideal divisor of $[c]$ in $K$ satisfies either $d=1$ or $2 \mid d$, then (3) has only the solution $(x, y, z)=$ $(a, 2,2)$. In this note we prove the following general result.

Theorem. If $b>8 \cdot 10^{6}, b \equiv \pm 5(\bmod 8)$ and $c$ is a prime power, then (3) has only the solution $(x, y, z)=(a, 2,2)$.

2. Preliminaries. For any $k \in \mathbb{N}$ with $k>1$ and $4 \nmid k$, let

$$
V(k)=\prod_{q \mid k}(1+\chi(q)),
$$

where $q$ runs over distinct prime factor of $k$,

$$
\chi(q)= \begin{cases}0 & \text { if } q=2 \\ (-1)^{(q-1) / 2} & \text { if } q \neq 2\end{cases}
$$

Supported by the National Natural Science Foundation of China. 
Lemma 1 ([1, Theorems $6 \cdot 7 \cdot 1$ and $6 \cdot 7 \cdot 4])$. The equation

$$
X_{1}^{2}+Y_{1}^{2}=k, \quad X_{1}, Y_{1} \in \mathbb{Z}, \operatorname{gcd}\left(X_{1}, Y_{1}\right)=1,
$$

has exactly $4 V(k)$ solutions $\left(X_{1}, Y_{1}\right)$.

Lemma 2 ([4, Chapter 15]). If $2 \nmid k$, then all solutions $(X, Y, Z)$ of the equation

$$
X^{2}+Y^{2}=k^{Z}, \quad X, Y, Z \in \mathbb{Z}, \operatorname{gcd}(X, Y)=1, Z>0,
$$

are given by

$Z \in \mathbb{N}, \quad X+Y \sqrt{-1}=\left(X_{1}+Y_{1} \sqrt{-1}\right)^{Z} \quad$ or $\quad Y+X \sqrt{-1}=\left(X_{1}+Y_{1} \sqrt{-1}\right)^{Z}$, where $\left(X_{1}, Y_{1}\right)$ runs over all solutions of (4).

Let $\alpha$ be a non-zero algebraic number with the defining polynomial $a_{0} z^{r}+a_{1} z^{r-1}+\ldots+a_{r}=a_{0}\left(z-\sigma_{1} \alpha\right) \ldots\left(z-\sigma_{r} \alpha\right) \in \mathbb{Z}[z]$, where $a_{0}>0$, $\sigma_{1} \alpha, \ldots, \sigma_{r} \alpha$ are all the conjugates of $\alpha$. Then

$$
h(\alpha)=\frac{1}{r}\left(\log a_{0}+\sum_{i=1}^{r} \log \max \left(1,\left|\sigma_{i} \alpha\right|\right)\right)
$$

is called Weil's height of $\alpha$.

Lemma 3 ([3, Section 10]). Let $\log \alpha$ be any non-zero determination of the logarithm of $\alpha$. If $r=2$ and $\Lambda=b_{1} \pi \sqrt{-1} / b_{2}-\log \alpha \neq 0$ for some $b_{1}, b_{2} \in \mathbb{Z}$ with $b_{1} b_{2} \neq 0$, then

$$
|\Lambda|>\exp \left(-20600 A(1.35+\log B+\log \log 2 B)^{2}\right),
$$

where $A=\max (1 / 2, h(\alpha)), B=\max \left(4,\left|b_{1}\right|,\left|b_{2}\right|\right)$.

Lemma 4. Let $X, Y \in \mathbb{Z}$ be such that $X Y \neq 0, \operatorname{gcd}(X, Y)=1$ and $2 \mid X Y$. Further, let $\varepsilon=X+Y \sqrt{-1}$ and $\bar{\varepsilon}=X-Y \sqrt{-1}$. If

$$
\left|\frac{\varepsilon^{n}-\bar{\varepsilon}^{n}}{\varepsilon-\bar{\varepsilon}}\right| \leq n
$$

for some $n \in \mathbb{N}$, then $n<8 \cdot 10^{6}$.

Proof. By much the same argument as in the proof of [2, Lemma 10], if (5) holds, then we have

(6) $\log n+\log |\varepsilon-\bar{\varepsilon}| \geq \log \left|\varepsilon^{n}-\bar{\varepsilon}^{n}\right| \geq n \log |\varepsilon|+\log \left|n \log \frac{\bar{\varepsilon}}{\varepsilon}-t \pi \sqrt{-1}\right|$, where $t \in \mathbb{Z}$ with $|t| \leq n$. Let $k=X^{2}+Y^{2}$ and $\Lambda=n \log (\bar{\varepsilon} / \varepsilon)-t \pi \sqrt{-1}$. Then $k \geq 5$ and $\bar{\varepsilon} / \varepsilon$ satisfies

$$
k\left(\frac{\bar{\varepsilon}}{\varepsilon}\right)^{2}-2\left(X^{2}-Y^{2}\right) \frac{\bar{\varepsilon}}{\varepsilon}+k=0, \quad \operatorname{gcd}\left(k, 2\left(X^{2}-Y^{2}\right)\right)=1 .
$$


This implies that $\bar{\varepsilon} / \varepsilon$ is not a root of unity and $h(\bar{\varepsilon} / \varepsilon)=\log \sqrt{k}$. Therefore, we have $\Lambda \neq 0$. Notice that $|\varepsilon|=\sqrt{k},|\varepsilon-\bar{\varepsilon}|<2 \sqrt{k}$, and the degree of $\bar{\varepsilon} / \varepsilon$ is equal to 2 . On applying Lemma 3 to (6), we get

$$
\log 2 \sqrt{k}+20600(\log \sqrt{k})(1.35+\log n+\log \log 2 n)^{2}>n \log \sqrt{k},
$$

whence we deduce that $n<8 \cdot 10^{6}$. The lemma is proved.

3. Proof of Theorem. Let $(x, y, z)$ be a solution of (3). If $2 \nmid y$, then from $(3)$ we get $(-b / c)=1$, where $(\cdot / \cdot)$ is Jacobi's symbol. Since $c \equiv 1$ $(\bmod 4)$ and $c \equiv 2 s^{2}(\bmod b)$ by $(2)$, if $b \equiv \pm 5(\bmod 8)$, then

$$
1=\left(\frac{-b}{c}\right)=\left(\frac{b}{c}\right)=\left(\frac{c}{b}\right)=\left(\frac{2 s^{2}}{b}\right)=\left(\frac{2}{b}\right)=-1,
$$

a contradiction. Similarly, we see from $(c / b)=-1$ that (3) has no solution $(x, y, z)$ with $2 \mid y$ and $2 \nmid z$. tion

If $2 \mid y$ and $2 \mid z$, then $(X, Y, Z)=\left(x, b^{y / 2}, z / 2\right)$ is a solution of the equa-

$$
X^{2}+Y^{2}=c^{2 Z}, \quad X, Y, Z \in \mathbb{Z}, \operatorname{gcd}(X, Y)=1, Z>0 .
$$

Since $c$ is a prime power and $c^{2}=a^{2}+b^{2}$, by Lemmas 1 and 2, we obtain the following four cases:

$$
\begin{array}{llll}
x+b^{y / 2} \sqrt{-1}=\lambda_{1}\left(a+\lambda_{2} b \sqrt{-1}\right)^{z / 2} & \text { or } & \lambda_{1}\left(b+\lambda_{2} a \sqrt{-1}\right)^{z / 2}, \\
b^{y / 2}+x \sqrt{-1}=\lambda_{1}\left(a+\lambda_{2} b \sqrt{-1}\right)^{z / 2} & \text { or } & \lambda_{1}\left(b+\lambda_{2} a \sqrt{-1}\right)^{z / 2},
\end{array}
$$

where $\lambda_{1}, \lambda_{2} \in\{-1,1\}$.

When $z=2$, we see from (7) that $x=a$ and $y=2$.

When $z>2$ and $2 \mid z / 2,(7)$ is impossible, since $a>1, b>1$ and $\operatorname{gcd}(a, b)=1$.

When $z>2$ and $2 \nmid z / 2$, we see from (7) that

$$
x+b^{y / 2} \sqrt{-1}=\lambda_{1}\left(a+\lambda_{2} b \sqrt{-1}\right)^{z / 2} .
$$

So we have

$$
\begin{aligned}
b^{y / 2-1}= & \lambda_{1} \lambda_{2}\left(\left(\begin{array}{c}
z / 2 \\
1
\end{array}\right) a^{z / 2-1}-\left(\begin{array}{c}
z / 2 \\
3
\end{array}\right) a^{z / 2-3}\left(-b^{2}\right)\right. \\
& \left.+\ldots+(-1)^{(z-2) / 4}\left(\begin{array}{c}
z / 2 \\
z / 2
\end{array}\right)\left(-b^{2}\right)^{(z-2) / 4}\right) \\
= & (-1)^{(z-2) / 4} \lambda_{1} \lambda_{2} \sum_{i=0}^{(z-2) / 4}(-1)^{i}\left(\begin{array}{c}
z / 2 \\
2 i
\end{array}\right) a^{2 i} b^{z / 2-2 i-1} .
\end{aligned}
$$


If $y=2$, then from (9) we get

$$
1=\sum_{i=0}^{(z-2) / 4}(-1)^{i}\left(\begin{array}{c}
z / 2 \\
2 i
\end{array}\right) a^{2 i} b^{z / 2-2 i-1},
$$

since $a^{2} \equiv 0(\bmod 4)$ and $b^{2} \equiv 1(\bmod 4)$. Let $2^{\alpha}\left\|a, 2^{\beta}\right\| b^{2}-1,2^{\gamma} \|(z-$ $2) / 4$ and $2^{\delta_{i}} \| 2 i$ for any $i \in \mathbb{N}$. Notice that $2 \| s t$ if $b \equiv \pm 5(\bmod 8)$ by $(2)$. We have $\alpha=2$ and $\beta=3$. Hence,

$$
2^{3+\gamma} \| b^{z / 2-1}-1 .
$$

On the other hand, since

$$
\delta_{i} \leq \frac{\log 2 i}{\log 2} \leq 2 i-1<2(2 i-1), \quad i \in \mathbb{N},
$$

we have

$$
\begin{aligned}
\left(\begin{array}{c}
z / 2 \\
2 i
\end{array}\right) a^{2 i}=\frac{a z}{2}\left(\frac{z-2}{2}\right)\left(\begin{array}{c}
z / 2-2 \\
2 i-2
\end{array}\right) \frac{a^{2 i-1}}{2 i(2 i-1)} & \equiv 0\left(\bmod 2^{4+\gamma}\right), \\
i & =1, \ldots,(z-2) / 4 .
\end{aligned}
$$

Therefore, we see from (11) and (12) that (10) is impossible.

If $y>2$, then $z / 2 \equiv 0(\bmod b)$ by $(9)$. Let $p$ be a prime factor of $b$. Further, let $p^{\alpha}\left\|b, p^{\beta}\right\| z / 2$ and $p^{\gamma_{i}} \| 2 i+1$ for any $i \in \mathbb{N}$. Notice that $2 \nmid b$, $p \geq 3$ and

We have

$$
\gamma_{i} \leq \frac{\log (2 i+1)}{\log p}<2 i, \quad i \in \mathbb{N}
$$

$$
\begin{array}{r}
\left(\begin{array}{c}
z / 2 \\
2 i+1
\end{array}\right) b^{2 i}=\frac{z}{2}\left(\begin{array}{c}
z / 2-1 \\
2 i
\end{array}\right) \frac{b^{2 i}}{2 i+1} \equiv 0\left(\bmod p^{\beta+1}\right) \\
i=1, \ldots,(z-2) / 4 .
\end{array}
$$

On applying (13) together with (9), we get

$$
\beta=\alpha\left(\frac{y}{2}-1\right) .
$$

Let $p$ run over distinct prime factors of $b$. We see from (14) that

$$
z / 2 \equiv 0\left(\bmod b^{y / 2-1}\right) .
$$

Recalling that $y>2$, we deduce from (15) that

$$
z / 2 \geq b^{y / 2-1} \geq b .
$$

Let $\varepsilon=a+b \sqrt{-1}$ and $\bar{\varepsilon}=a-b \sqrt{-1}$. From (8) and (9), we get

$$
\left|\frac{\varepsilon^{z / 2}-\bar{\varepsilon}^{z / 2}}{\varepsilon-\bar{\varepsilon}}\right|=b^{y / 2-1} \text {. }
$$


By (16), on applying Lemma 4 to (17), we obtain $z / 2<8 \cdot 10^{6}$. Thus, by (16), we deduce $b<8 \cdot 10^{6}$. The Theorem is proved.

\section{References}

[1] L.-K. Hua, Introduction to Number Theory, Springer, Berlin, 1982.

[2] M.-H. Le, The diophantine equation $x^{2}+D^{m}=p^{n}$, Acta Arith. 52 (1989), 255-265.

[3] M. Mignotte and M. Waldschmidt, Linear forms in two logarithms and Schneider's method, III, Ann. Fac. Sci. Toulouse Math. 97 (1989), 43-75.

[4] L. J. Mordell, Diophantine Equations, Academic Press, London, 1969.

[5] N. Terai, The Diophantine equation $x^{2}+q^{m}=p^{n}$, Acta Arith. 63 (1993), 351-358.

DEPARTMENT OF MATHEMATICS

ZHANJIANG TEACHER'S COLLEGE

P.O. BOX 524048

ZHANJIANG, GUANDONG

P.R. CHINA 